\title{
Association of Serum Uric Acid Levels with Components of Metabolic Syndrome: A Cross-Sectional Analysis in a Saudi Adult Population
}

\author{
Manal S. Fawzy ${ }^{1,2 *}$; Baraah T. Abu AlSel ${ }^{3}$ \\ ${ }^{1}$ Department of Biochemistry, Faculty of Medicine, Northern Border University, Arar, Saudi Arabia \\ ${ }^{2}$ Department of Medical Biochemistry and Molecular Biology, Faculty of Medicine, Suez Canal University, \\ Ismailia, Egypt \\ ${ }^{3}$ Department of Microbiology, Northern Border University, Arar, Saudi Arabia
}

\begin{abstract}
Background: This study aimed to investigate the possible relations between serum uric acid (UA) and metabolic syndrome (MetS) and its components in a Saudi adult population sample.

Methods and Results: This cross-sectional study enrolled consecutive adult MetS and non-MetS subjects (90 subjects in each group). Glycemic control indices (fasting blood sugar (FBS), HbAlc, insulin, HOMA-IR index), lipid profile/ratios, and renal function tests were also evaluated.

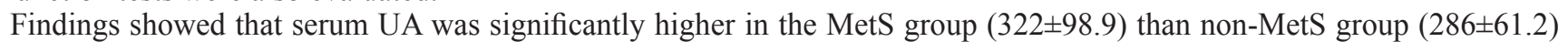
$\mu \mathrm{mol} / \mathrm{L}$. The fourth quartile of serum UA showed a higher frequency of MetS (73.3\%) and central obesity (82.2\%), and higher mean values of triglycerides and FBS as well as lower mean values for HDL-C relative to the first quartiles. Data stratification by sex showed consistent associations of BMI, abdominal obesity, HDL-C, TG/HDL-C, and serum creatinine levels with serum UA in both men and women. Serum UA at $310 \mu \mathrm{mol} / \mathrm{L}$ concentration might be a good predictor for MetS/its components in men. In contrast, at a cut-off level of $275.0 \mu \mathrm{mol} / \mathrm{L}$, it could significantly predict only obesity and high FBS in women.

Conclusion: Serum UA levels are associated with MetS and may predict MetS and/or its components at specific levels in a sex-dependent pattern in the study population. (International Journal of Biomedicine. 2020;10(4):457-466.)
\end{abstract}

Key Words: metabolic syndrome $\bullet$ uric acid $\bullet$ lipid profile $\bullet$ insulin resistance $\bullet$ Saudi adults

\section{Abbreviations}

AUC, area under curve; BMI, body mass index; BP, blood pressure; BUN, blood urea nitrogen; CRE, serum creatinine; FBS, fasting blood sugar; HbA1c, glycated hemoglobin; HDL-C, high-density lipoprotein cholesterol; HOMA-IR, Homeostasis Model Assessment - IR index; IDL-C, intermediate-density lipoprotein cholesterol; IR, insulin resistance; IGT, impaired glucose tolerance; LDL, low-density lipoprotein; LDL-C, low-density lipoprotein cholesterol; MetS, metabolic syndrome; TC, total cholesterol; TG, triglycerides; UA, uric acid; VLDL-C, very low-density lipoprotein cholesterol; WC, waist circumference

\section{Introduction}

Uric acid (2,6,8-trioxypurine) is the final oxidation product of purine metabolism. The formation of uric acid (UA) is through the enzyme xanthine oxidase, which oxidizes

*Corresponding author: Prof. Manal S. Fawzy, Department of Medical Biochemistry, Faculty of Medicine, Northern Border University, Saudi Arabia. E-mails: manal2_khashana@ymail.com, manal.darwish@nbu.edu.sa oxypurines (xanthine and hypoxanthine) (https://druginfo. nlm.nih.gov/m.drugportal/rn/69-93-2). For a long time, UA was just considered as a risk for the development of gout and kidney stones. ${ }^{(1)}$ Since the 1900 s, however, the accumulated evidence, based mainly on epidemiological studies, has linked serum UA levels to metabolic syndrome (MetS), chronic kidney disease, and cardio-cerebrovascular events. ${ }^{(2-4)} \mathrm{A}$ growing body of evidence has suggested that UA may not only be considered as a risk factor of MetS but also an independent predictor of cardio-metabolic diseases and mortality. ${ }^{(1,5,6)}$ 
Moreover, in animal models, decreasing UA levels can prevent or reverse features of MetS. ${ }^{(7,8)}$

Although UA can function as an antioxidant, it might be an inexpensive marker of the effects of oxidative stress because its antioxidant activity can be overcome by the pro-oxidant and pro-inflammatory effects on cells..$^{(1,5)}$ In experiments with cultured vascular cells, UA induces cellular proliferation, inflammation, oxidative stress, and the activation of the local renin-angiotensin system. ${ }^{(1)}$

In MetS, hyperuricemia has been assigned to hyperinsulinemia and to decline in uric acid excretion associated with kidney dysfunction and is not acknowledged as the main mediator of metabolic syndrome, renal disease, and cardiovascular disorder development. However, more recent investigations have altered this traditional view and shown by providing compelling evidence to support an independent link between hyperuricemia and increased risk of MetS, diabetes, hypertension, kidney disease, and cardiovascular disorders. Despite these emerging findings, controversy regarding the exact role of uric acid in inducing these diseases remains to be uncovered. ${ }^{(6,9)}$ Interestingly, we have previously identified a high rate of obesity and type $2 \mathrm{DM}$ with their complications. ${ }^{(10,11)}$ In this sense, this study aimed to investigate the possible relations between serum uric acid (UA) and metabolic syndrome (MetS) and its components in a Saudi adult population sample.

\section{Subjects and Methods}

\section{Study population}

A total of 180 Saudi adult non-smoker participants aged between 24 and 70 years, presenting at the General Central Hospital, were recruited between June 2017 and December 2017 for this study. All participants were allocated into 2 study groups. The MetS group included 90 individuals with MetS; the non-MetS group included 90 individuals without MetS. MetS was defined according to the revised version of the third report of the National Cholesterol Education Program Adult Treatment Panel III (NCEP ATP III), ${ }^{(12)}$ i.e. having any 3 of the following 5 diagnostic criteria: (i) Elevated WC ( $\geq 92 \mathrm{~cm}$ in men and $\geq 87$ $\mathrm{cm}$ in women based on the Saudi Abnormal Glucose Metabolism and Diabetes Impact Study (SAUDI-DM); ${ }^{(13)}$ (ii) Elevated TG ( $\geq 1.7 \mathrm{mmol} / \mathrm{L}$ or on drug treatment for elevated TG; (iii) Reduced HDL-C $(\leq 1.03 \mathrm{mmol} / \mathrm{L}$ in men and $\leq 1.3 \mathrm{mmol} / \mathrm{L}$ in women or on drug treatment for reduced HDL-C); (iv) Elevated BP (SBP $\geq 130 \mathrm{mmHg}$ or DBP $\geq 85 \mathrm{mmHg}$ or on antihypertensive drug treatment in a patient with a history of hypertension); (v) Elevated fasting glucose $(\geq 5.6 \mathrm{mmol} / \mathrm{L})$ or on drug treatment for elevated glucose. ${ }^{(14,15)}$ Exclusion criteria were participants aged $<18$ years, pregnancy or breastfeeding, subjects with a history of chronic disease (cardiovascular disease, cancer, stroke, kidney diseases, blood disorders, and gout), treatment with drugs that can affect the results of the study.

Trained nurses through the respective hospital were specified for questionnaire data filling (e.g. age, sex, history of diabetes, hypertension, and dyslipidemia, etc.), anthropometric measurements (height, weight, WC) using Digital Pearson Scale (ADAM Equipment Inc., USA), BP measurement (3 times by using a mercury blood pressure device after the subjects had rested longer than $5 \mathrm{~min}),{ }^{(16)}$ and blood sampling following standard protocols to ensure accurate and complete demographic and clinical information for each included participant.

This study was checked against the STROBE (Strengthening the Reporting of OBservational Studies in Epidemiology) checklist and conducted following the "ethical standards of the institutional and national research committee" and with the Helsinki Declaration and its later amendments or comparable ethical standard. It was reviewed and approved by the Medical and Bioethics local committee. Each participant signed informed consent before taking part.

\section{Sample collection and the biochemical analysis}

Overnight fast venous blood samples were collected in plain tubes $(5 \mathrm{~mL})$ for centrifugation $(2500 \mathrm{rpm} \times 15$ minutes $)$ and EDTA tubes $(2 \mathrm{~mL})$ for automated glycated hemoglobin (HbA1c) estimation (COBAS, INTEGRA, Roche Diagnostics, USA). The separated sera were divided into aliquots and stored at $-80{ }^{\circ} \mathrm{C}$ until the time of biochemical analysis. Routine laboratory measurements, including blood urea nitrogen (BUN), serum creatinine (CRE), serum uric acid (UA), fasting blood sugar (FBS), and lipid profile (i.e. TC, HDL-C, LDL-C and TG) were done using commercially available kits on Cobas Integra 400 plus Biochemical analyzer (Roche Diagnostics)". The ratios TC/HDL-C and TG/HDL-C which indicate "the balance between all atherogenic cholesterols (VLDL-C, IDL-C, and LDL-C), and antiatherogenic cholesterol (HDL-C)", were calculated as determinants of cardiovascular risk.(17) NonHDL-C (i.e. TC - HDL-C) was also calculated as a valuable predictor of premature atherosclerosis and coronary events. ${ }^{(18)}$

Serum insulin was measured by "Electrochemiluminescence Immunoassay (Cobas, Roche Diagnostics, USA)" according to the instructions recommended by the manufacturer. Insulin resistance was assessed using HOMA-IR. The calculation formula was as follows: HOMA-IR=(fasting insulin $[\mu \mathrm{IU} / \mathrm{mL}] \times$ fasting glucose $[\mathrm{mmol} / \mathrm{L}]) / 22.5{ }^{(19,20)}$

All the quality control measurements were followed during the laboratory work including running the appropriate calibrators and controls before each run to ensure the performance of the assay.

\section{Statistical analysis}

All patient data were coded and anonymized before the analysis. The normally distributed continuous values (i.e. checked by Kolmogorov-Smirnov test) were expressed as mean \pm standard deviation (SD) and compared using the Student's t-test (for two groups). One-way analysis of variance (ANOVA) on a rank test ( $>$ two groups) followed by Bonferroni multiple comparison tests were also applied. Categorical variables were presented as frequencies (percentages) and compared by Chisquare or Fisher's exact tests. Moreover, Pearson's correlation coefficient was used to test correlations between serum UA and other study variables. Logistic regression analysis was applied to calculate the odds ratios (OR) and $95 \%$ confidence intervals (CI) for variables in the study groups, which were adjusted for significant confounding factors as age, parameters of glycemic control, lipid profile, and kidney function test. Receiver operating characteristics (ROC) analysis was used to calculate the area 
under the curve (AUC) for serum UA and to find the best cutoff values associated with maximum sensitivity and specificity to identify MetS and its components. Calculation of the study power using "G power-3 software version 3.0.10 (http://www. gpower.hhu.de/)", showed that with the specified study design, and allowable error rate; alpha error $=0.05$ with sample size 90 for each group can give $89 \%$ power with nearly an effect size $=0.44$. The optimal cutoff value for each clinical-laboratory measurement to predict MetS was calculated. Results with $P<0.05$ were considered statistically significant. Data analysis was done by the Statistical Package for the Social Sciences software (IBM SPSS Statistics for Windows, Version 22.0. Armonk, NY).

\section{Results}

\section{Baseline characteristics of study participants}

The baseline clinical and biochemical characteristics of the study subjects were summarized in Tables 1 and 2. Also, the associations of these parameters with MetS components were presented. As shown in Table 1, individuals older than 45 years had higher odds ( $\mathrm{OR}=3.28 ; 95 \%$ CI: $1.76-6.13)$ of having MetS compared with those less than 45 years old. The odds ratios of central obesity, hypertension, diabetes mellitus, hyperglycemia, dyslipidemia, hypercholesterolemia, hypertriglyceridemia, elevated LDL-C, and increased IR were $5.61,27.9,5.20,4.33,11.2,2.21,14.8,4.37$, and 2.91, respectively, (all $P<0.05$ ) for risk to develop MetS relative to ones who do not have any of these disorders. As expected, subjects in the MetS group had significantly higher values of glycemic parameters (FBG, HbAlc, insulin, and HOMA-IR index), lipid profile and ratios (TC, TG, LDL-C, TC/HDL-C, TG/HDL-C, and Non-HDL-C), BUN, and UA than ndividuals in the non-MetS group (Table 2).

\section{Prevalence of MetS components among the study participants}

Of the 180 study subjects, central obesity was observed in $112(62 \%)$ of participants in whom women had 3.8 times susceptibility for getting this MetS component than men (95\% CI: $1.96-7.54 ; P<0.001)$. In contrast, low HDL-C levels were more prominent in men $(64 \%)$ than women $(32 \%)$. Other MetS components (i.e. hypertension, hypertriglyceridemia, and IGT) show consistency among men and women (Table 3).

Table 1.

Clinical and biochemical characteristics of the study participants

\begin{tabular}{|c|c|c|c|c|c|}
\hline Variables & & Non-MetS & MetS & $P$ & OR $(95 \% \mathrm{CI})$ \\
\hline Number & & $(\mathrm{n}=90)$ & $(\mathrm{n}=90)$ & & \\
\hline \multirow{3}{*}{ Age } & Mean \pm SD & $37.3 \pm 16.3$ & $43.1 \pm 12.0$ & $0.004^{b}$ & \\
\hline & $\leq 45$ years & $66(73.3)$ & $41(45.6)$ & $<0.001^{{ }^{a}}$ & Reference \\
\hline & $>45$ years & $24(26.7)$ & $49(54.4)$ & & $3.28(1.76-6.13)$ \\
\hline \multirow{2}{*}{ Sex } & Male & $23(25.6)$ & $30(33.3)$ & $0.252^{a}$ & $0.68(0.36-1.30)$ \\
\hline & Female & $67(74.4)$ & $60(66.7)$ & & \\
\hline Weight, kg & Mean \pm SD & $79.3 \pm 16.3$ & $90.8 \pm 19.1$ & $<0.001^{\mathrm{b}}$ & \\
\hline Height, $\mathrm{cm}$ & Mean \pm SD & $160.3 \pm 8.4$ & $163.3 \pm 9.17$ & $0.025^{b}$ & \\
\hline BMI, $\mathrm{kg} / \mathrm{m}^{2}$ & Mean \pm SD & $30.9 \pm 6.4$ & $33.9 \pm 6.6$ & $0.001^{\mathrm{b}}$ & \\
\hline \multirow{2}{*}{ Abdominal obesity } & Negative & $51(56.7)$ & $17(18.9)$ & $<0.001^{\text {a }}$ & Reference \\
\hline & Positive & $39(43.3)$ & $73(81.1)$ & & $5.61(2.86-11.0)$ \\
\hline \multirow{2}{*}{ Hypertension } & Negative & $78(86.7)$ & $17(18.9)$ & $<0.001^{a}$ & Reference \\
\hline & Positive & $12(13.3)$ & $73(81.1)$ & & $27.9(12.4-62.4)$ \\
\hline \multirow{2}{*}{ Diabetes mellitus } & Negative & $78(86.7)$ & $50(55.6)$ & $<0.001^{a}$ & Reference \\
\hline & Positive & $12(13.3)$ & $40(44.4)$ & & $5.20(2.49-10.85)$ \\
\hline \multirow{2}{*}{ High FBS } & Negative & $78(86.7)$ & $54(60.0)$ & $<0.001^{a}$ & Reference \\
\hline & Positive & $12(13.3)$ & $36(40.0)$ & & $4.33(2.06-9.08)$ \\
\hline \multirow{2}{*}{ High insulin } & Negative & $81(90.0)$ & $74(82.2)$ & $0.195^{\mathrm{a}}$ & Reference \\
\hline & Positive & $9(10.0)$ & $16(17.8)$ & & $1.94(0.81-4.67)$ \\
\hline \multirow{2}{*}{ Dyslipidemia } & Negative & $25(27.8)$ & $3(3.3)$ & $<0.001^{a}$ & Reference \\
\hline & Positive & $65(72.2)$ & $87(96.7)$ & & $11.15(3.22-38.5)$ \\
\hline \multirow{2}{*}{ High TC } & Negative & $62(68.9)$ & $45(50.0)$ & $0.015^{\mathrm{a}}$ & Reference \\
\hline & Positive & $28(31.1)$ & $45(50.0)$ & & $2.21(1.20-4.06)$ \\
\hline \multirow{2}{*}{ High TG } & Negative & $85(94.4)$ & $48(53.3)$ & $<0.001^{a}$ & Reference \\
\hline & Positive & $5(5.6)$ & $42(46.7)$ & & $14.8(5.51-40.1)$ \\
\hline \multirow{2}{*}{ High LDL-c } & Negative & $42(46.7)$ & $15(16.7)$ & $<0.001^{a}$ & Reference \\
\hline & Positive & $48(53.3)$ & $75(73.3)$ & & $4.37(2.19-8.74)$ \\
\hline \multirow{2}{*}{ Low HDL-c } & Negative & $52(57.8)$ & $53(58.9)$ & $0.880^{\mathrm{a}}$ & Reference \\
\hline & Positive & $38(42.2)$ & $37(41.1)$ & & $0.95(0.52-1.72)$ \\
\hline \multirow{2}{*}{ HOMA-IR } & IS & $79(87.8)$ & $64(71.2)$ & $0.009^{\mathrm{a}}$ & Reference \\
\hline & IR & $11(12.2)$ & $26(28.8)$ & & $2.91(1.34-6.35)$ \\
\hline
\end{tabular}

${ }^{a}$ Fisher's Exact or Chi-square tests were used for qualitative variables, and ${ }^{b}$ Student's $t$-test was used for quantitative data. According to HOMA-IR all participants were divided into insulin-sensitive (IS) and insulin resistance (IR, >1.9) groups (Vogeser et al., 2007). 
Table 2.

Biochemical characteristics of the study participants

\begin{tabular}{|l|c|c|c|}
\hline \multicolumn{1}{|c|}{ Variables } & Non-MetS $(\mathrm{n}=90)$ & MetS $(\mathrm{n}=90)$ & $P$ \\
\hline FBS, $\mathrm{mmol} / \mathrm{L}$ & $5.6 \pm 1.9$ & $6.8 \pm 3.1$ & 0.002 \\
\hline $\mathrm{HbAlc}, \%$ & $5.36 \pm 1.1$ & $6.7 \pm 1.65$ & $<0.001$ \\
\hline Insulin, $\mathrm{mlU} / \mathrm{mL}$ & $4.49 \pm 2.7$ & $5.82 \pm 3.8$ & 0.008 \\
\hline HOMA-IR & $1.15 \pm 0.9$ & $1.6 \pm 1.0$ & 0.001 \\
\hline TC, mmol/L & $4.7 \pm 1.09$ & $5.2 \pm 0.86$ & 0.002 \\
\hline TG, $\mathrm{mmol} / \mathrm{L}$ & $1.14 \pm 0.4$ & $1.68 \pm 0.9$ & $<0.001$ \\
\hline LDL-C, mmol/L & $2.9 \pm 1.0$ & $3.3 \pm 0.8$ & 0.003 \\
\hline HDL-C, mmol/L & $1.22 \pm 0.3$ & $1.19 \pm 0.3$ & 0.583 \\
\hline TC/HDL-C & $4.11 \pm 1.4$ & $4.69 \pm 1.4$ & 0.009 \\
\hline TG/HDL-C & $1.0 \pm 0.52$ & $1.5 \pm 1.07$ & $<0.001$ \\
\hline Non-HDL-C & $3.57 \pm 1.1$ & $4.05 \pm 0.9$ & 0.002 \\
\hline BUN, mmol/L & $5.13 \pm 1.9$ & $6.5 \pm 2.2$ & $<0.001$ \\
\hline CRE, mmol/L & $64.1 \pm 14.8$ & $67.4 \pm 16.0$ & 0.148 \\
\hline UA, $\mathrm{mmol} / \mathrm{L}$ & $286 \pm 61.2$ & $322 \pm 98.9$ & 0.004 \\
\hline
\end{tabular}

Table 3.

Prevalence of components of metabolic syndrome among the study participants

\begin{tabular}{|l|c|c|c|c|c|}
\hline \multicolumn{1}{|c|}{ Component } & $\begin{array}{c}\text { Total } \\
(\mathrm{n}=180)\end{array}$ & $\begin{array}{c}\text { Men } \\
(\mathrm{n}=53)\end{array}$ & $\begin{array}{c}\text { Women } \\
(\mathrm{n}=127)\end{array}$ & $P$ & ${ }^{\mathrm{a}} \mathrm{OR}(95 \% \mathrm{CI})$ \\
\hline Central obesity & 112 & $21(39.6)$ & $91(71.1)$ & $<0.001$ & $3.85(1.96-7.54)$ \\
\hline Hypertension & 85 & $29(54.7)$ & $56(44.1)$ & 0.251 & $0.65(0.34-1.24)$ \\
\hline High TG & 47 & $17(32.1)$ & $30(23.6)$ & 0.266 & $0.65(0.32-1.32)$ \\
\hline Low HDL-C & 75 & $34(64.2)$ & $41(32.3)$ & $<0.001$ & $0.26(0.13-0.52)$ \\
\hline IGT & 52 & $16(30.2)$ & $36(28.3)$ & 0.857 & $0.91(0.45-1.84)$ \\
\hline
\end{tabular}

${ }^{a}$ OR (95\% CI): adjusted odds ratio for age (95\% confidence interval).

\section{Prevalence of MetS and its components among serum UA quartiles}

Figure 1 shows the prevalence of MetS and its components among the four quartiles [Q1: $<248.3$, Q2: 248.3- $<290$, Q3: 290- <361.7, Q4: $\geq 361.7)$ of serum UA.
(A)

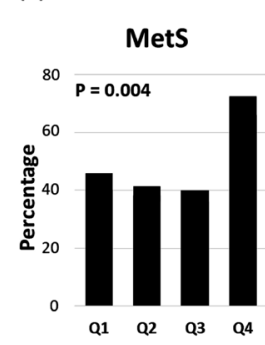

Serum UA quartiles

(D)

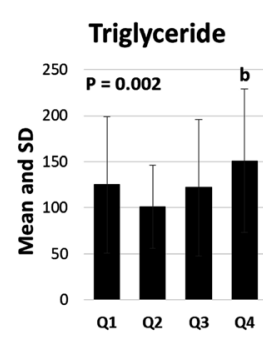

Serum UA quartiles
(B)

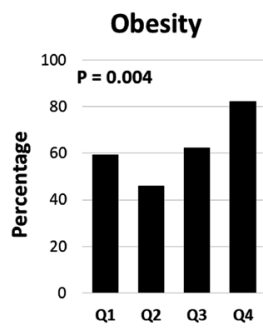

Serum UA quartiles

(E)

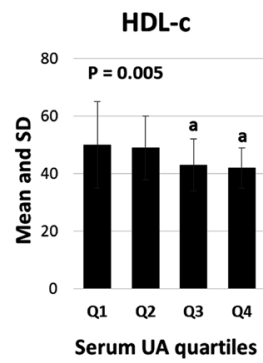

(c)

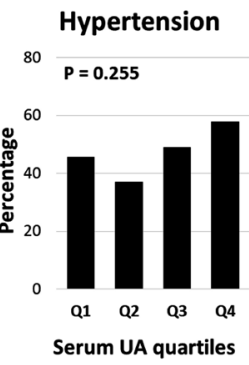

(F)

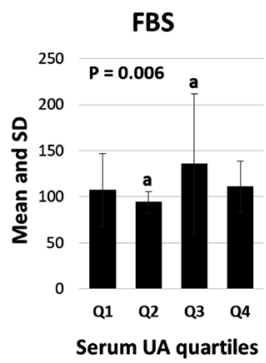

Fig. 1. Overall analysis of the prevalence of MetS and its components by quartiles (Q1-Q4) of serum UA. Data are presented as percentages $(A-C)$ or mean and $S D(D-F)$.
Overall, individuals in the Q4 of serum UA showed a significantly higher frequency of MetS (73.3\%) and central obesity $(82.2 \%)$ relative to the ones in the Q1-Q3 (UA-Q1 to UA-Q3; $45.5 \%, 41.3 \%, 40.0 \%$ of MetS frequency, and $59.1 \%$, $45.7 \%$, and $62.6 \%$ of central obesity frequency, respectively).

Furthermore, higher mean values of TG and FBS and lower mean values for HDL-C were observed in UA-Q4 relative to the first ones (Table 4). Data stratification by sex showed a central obesity prevalence increase in UA-Q4 for both males and females. At the same time, subjects with higher mean TG levels and lower mean HDL-C levels were more frequent in UA-Q4 of men relative to that in women (Table 4).

\section{Association between serum $U A$ and the clinical-laboratory parameters}

Correlations between serum UA and the clinicallaboratory parameters are summarized in Table 5. Overall analysis showed that serum UA was correlated with age $(\mathrm{r}=0.174, \quad P=0.019)$, weight $(\mathrm{r}=0.318, \quad P<0.001)$, height ( $\mathrm{r}=0.236, P=0.001)$, BMI $(\mathrm{r}=0.175, P=0.018)$ and abdominal obesity ( $\mathrm{r}=0.216, P=0.004)$. In the current study, we found an inverse correlation between serum UA and HDL-C levels ( $\mathrm{r}=-$ $0.259, P<0.001)$ and significant positive correlations between serum UA and other laboratory parameters, including the glycemic variables (FBS, HbAlc, insulin, and HOMA-IR), the lipid profile-related variables and ratios (TC/HDL-C, TG/ HDL-C, and non-HDL-C), and kidney function test-related variables (BUN and CRE) as shown in Table 5. Interestingly, data stratification by sex showed consistent associations of BMI, abdominal obesity, HDL-C, TG/HDL-C, and serum creatinine levels with serum UA in both men and women.

\section{Evaluation of serum $U A$ concentration and other clinical- laboratory parameters in diagnosing MetS and its components}

An overall analysis has revealed that serum UA at a cut-off point of $295.05 \mu \mathrm{mol} / \mathrm{L}$ could predict MetS, central obesity, high levels of TG, and low HDL-C in the total study participants (Figure 2).
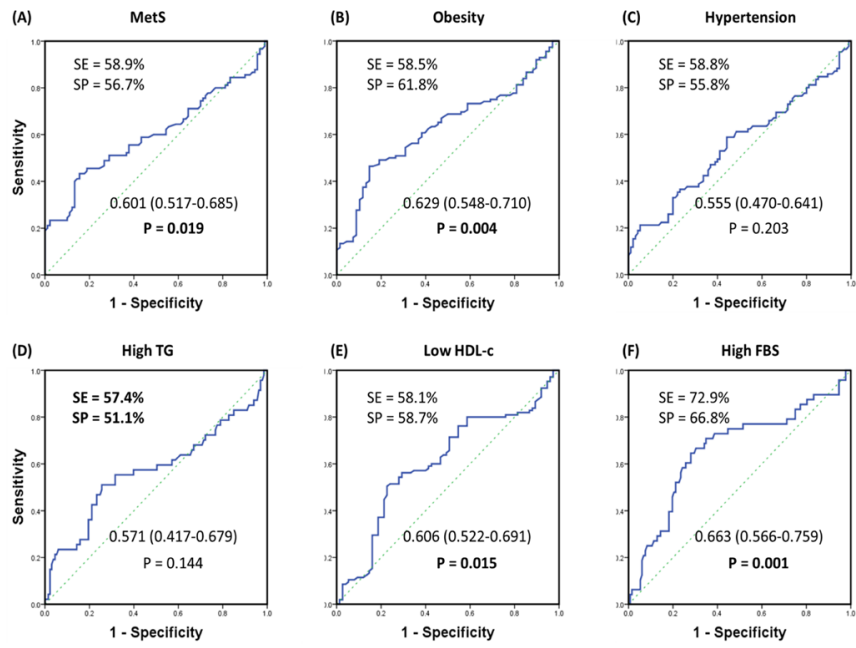

Fig. 2. An overall analysis of the ROC curve for serum UA concentration predicting MetS and its components. (A) Metabolic syndrome, (B) Central obesity, (C) Hypertension, (D) Hypertriglyceridemia, (E) Low HDL-C, (F) High FBS. 
Table 4.

Prevalence of MetS and its components by quartiles (Q1-Q4) of serum UA stratified by sex

\begin{tabular}{|c|c|c|c|c|c|}
\hline Component & UA-Q1 & UA-Q2 & UA-Q3 & UA-Q4 & $P$ \\
\hline \multicolumn{6}{|l|}{ Overall } \\
\hline MetS & $20(45.5)$ & $19(41.3)$ & $18(40.0)$ & $33(73.3)$ & 0.004 \\
\hline Central obesity & $26(59.1)$ & $21(45.7)$ & $28(62.2)$ & $37(82.2)$ & 0.004 \\
\hline Hypertension & $20(45.5)$ & $17(37.0)$ & $22(48.9)$ & $26(57.8)$ & 0.255 \\
\hline TG & $1.4 \pm 0.8$ & $1.14 \pm 0.5$ & $1.4 \pm 0.8$ & $1.7 \pm 0.9^{b}$ & 0.002 \\
\hline HDL-C & $1.3 \pm 0.4$ & $1.3 \pm 0.3$ & $1.1 \pm 0.2^{\mathrm{a}}$ & $1.1 \pm 0.2^{\mathrm{a}}$ & 0.005 \\
\hline FBS & $6.0 \pm 2.2$ & $5.2 \pm 0.7^{\mathrm{a}}$ & $7.6 \pm 4.2^{\mathrm{a}}$ & $6.2 \pm 1.6$ & 0.006 \\
\hline \multicolumn{6}{|l|}{ Men $(n=53)$} \\
\hline MetS & $4(57.1)$ & $4(40.0)$ & $8(44.4)$ & $14(77.8)$ & 0.139 \\
\hline Central obesity & $2(28.6)$ & $0(0.0)$ & $8(44.4)$ & $11(61.1)$ & 0.014 \\
\hline Hypertension & $2(28.6)$ & $5(50.0)$ & $11(61.1)$ & $11(61.1)$ & 0.455 \\
\hline TG & $1.3 \pm 0.5$ & $0.9 \pm 0.4$ & $1.5 \pm 90.8$ & $2.0 \pm 1.1$ & 0.008 \\
\hline HDL-C & $1.3 \pm 0.2$ & $1.4 \pm 0.1$ & $1.1 \pm 0.2$ & $1.1 \pm 0.2$ & 0.002 \\
\hline FBS & $5.4 \pm 1.0$ & $5.4 \pm 0.6$ & $9.6 \pm 5.8$ & $5.9 \pm 1.3$ & 0.359 \\
\hline \multicolumn{6}{|l|}{ Women $(n=127)$} \\
\hline MetS & $16(43.2)$ & $15(41.7)$ & $10(37.0)$ & $19(70.4)$ & 0.055 \\
\hline Central obesity & $24(64.9)$ & $21(58.3)$ & $20(74.1)$ & $26(96.3)$ & 0.007 \\
\hline Hypertension & $18(48.6)$ & $12(33.3)$ & $11(40.7)$ & $15(55.6)$ & 0.313 \\
\hline TG & $1.4 \pm 0.9$ & $1.2 \pm 0.5$ & $1.3 \pm 0.7$ & $1.5 \pm 0.6$ & 0.107 \\
\hline HDL-C & $1.3 \pm 0.4$ & $1.2 \pm 0.3$ & $1.1 \pm 0.3$ & $1.1 \pm 0.2$ & 0.084 \\
\hline FBS & $6.1 \pm 2.4$ & $5.2 \pm 0.7$ & $6.2 \pm 1.9$ & $6.4 \pm 1.8$ & 0.006 \\
\hline
\end{tabular}

Data are presented as frequency (percentage) or mean and SD. Pearson Chi-square and Kruskal-Wallis tests were applied, followed by Bonferroni multiple comparison test. a: statistically significant relative to UA-Q1, b: statistically significant relative to UA-Q2.

Table 5.

Correlation analysis between serum UA level and the clinicallaboratory parameters

\begin{tabular}{|c|c|c|c|}
\hline Clinical data & Total subjects & Men & Women \\
\hline Age, years & $0.174(0.019)$ & $-0.056(0.693)$ & $0.230(0.009)$ \\
\hline Weight, kg & $0.318(<0.001)$ & $0.555(<0.001)$ & $0.274(0.002)$ \\
\hline Height, cm & $0.236(0.001)$ & $0.312(0.023)$ & $0.083(0.354)$ \\
\hline BMI, kg/m² & $0.175(0.018)$ & $0.495(<0.001)$ & $0.178(0.046)$ \\
\hline Abdominal obesity & $0.216(0.004)$ & $0.446(0.001)$ & $0.249(0.005)$ \\
\hline Hypertension & $0.095(0.204)$ & $0.235(0.090)$ & $0.006(0.946)$ \\
\hline Diabetes & $0.122(0.104)$ & $0.071(0.612)$ & $0.114(0.200)$ \\
\hline Dyslipidemia & $0.063(0.402)$ & $0.006(0.963)$ & $0.139(0.118)$ \\
\hline \multicolumn{4}{|l|}{ Laboratory tests } \\
\hline FBS, mmol/L & $0.221(0.003)$ & $0.119(0.395)$ & $0.260(0.003)$ \\
\hline HbAlc, $\%$ & $0.243(0.001)$ & $0.025(0.858)$ & $0.343(<0.001)$ \\
\hline Insulin, mlU/mL & $0.169(0.023)$ & $0.361(0.008)$ & $0.088(0.325)$ \\
\hline HOMA-IR & $0.246(0.001)$ & $0.392(0.004)$ & $0.147(0.100)$ \\
\hline $\mathrm{TC}, \mathrm{mmol} / \mathrm{L}$ & $0.094(0.207)$ & $0.439(0.001)$ & $-0.004(0.964)$ \\
\hline $\mathrm{TG}, \mathrm{mmol} / \mathrm{L}$ & $0.243(0.001)$ & $0.432(0.001)$ & $0.148(0.097)$ \\
\hline LDL-C, mmol/L & $0.122(0.103)$ & $0.380(0.005)$ & $0.059(0.510)$ \\
\hline HDL-C, mmol/L & $-0.259(<0.001)$ & $-0.459(0.001)$ & $-0.200(0.024)$ \\
\hline TC/HDL-C & $0.243(0.001)$ & $0.481(<0.001)$ & $0.165(0.063)$ \\
\hline TG/HDL-C & $0.299(<0.001)$ & $0.464(<0.001)$ & $0.213(0.016)$ \\
\hline Non-HDL-C & $0.167(0.025)$ & $0.467(<0.001)$ & $0.071(0.427)$ \\
\hline $\mathrm{BUN}, \mathrm{mmol} / \mathrm{L}$ & $0.211(0.004)$ & $0.049(0.726)$ & $0.210(0.018)$ \\
\hline $\mathrm{CRE}, \mathrm{mmol} / \mathrm{L}$ & $0.502(<0.001)$ & $0.646(<0.001)$ & $0.427(<0.001)$ \\
\hline
\end{tabular}

Data are presented as the correlation coefficient ( $P$-values). Abdominal obesity: $W C \geq 92 \mathrm{~cm}$ in men and $\geq 87 \mathrm{~cm}$ in women.
On stratified analysis by sex, serum UA at approximately $310 \mu \mathrm{mol} / \mathrm{L}$ concentration was found to be a good predictor for MetS and all its components in men. While at a cut-off level of $275.0 \mu \mathrm{mol} / \mathrm{L}$, serum UA could significantly predict obesity and high FBS among all MetS components in women [AUC (95\%CI): 0.659 (0.566-0.753), and 0.691 (0.522-0.831), respectively] (Table 6).

On investigating the optimal cutoff values of the clinical-laboratory parameters in the prediction of MetS, the BMI was the best anthropometric measurement to predict MetS [AUC (95\% CI): 0.689 (0.609-0.769)] with sensitivity $(80 \%)$ and specificity $(60 \%)$ which moderately minimizes the false-positive/-negative cases. The positive likelihood ratio (PLR) was equivalent to 2.0, suggesting that those subjects with $\mathrm{BMI} \geq 30.4 \mathrm{~kg} / \mathrm{m}^{2}$ may present approximately twice the chance of a positive diagnosis being true. In contrast, the negative likelihood ratio (NLR) corresponded to 0.33 , which is close to three times the chance of a negative diagnosis confirming the absence of MetS. The PLR and NLR of UA were 1.36 and 0.72 , respectively, at $295 \mu \mathrm{mol} / \mathrm{L}$ serum level (Table 7).

\section{Discussion}

Given the growing prevalence of obesity, prediabetes, and diabetes, ${ }^{(21,22)}$ the study of factors that interplay with MetS and/or its components becomes an essential area of public health concern. 
Table 6.

Evaluation of serum UA concentration in diagnosing MetS and its components stratified by sex

\begin{tabular}{|c|c|c|c|c|c|c|c|c|c|}
\hline Variable & AUC & $95 \% \mathrm{CI}$ & $\mathrm{S}$ & $P$ & $\begin{array}{l}\text { Cut-off } \\
(\mathrm{mmol} / \mathrm{L})\end{array}$ & SE & SP & $\mathrm{LR}+$ & LR- \\
\hline \multicolumn{10}{|l|}{ OVERALL } \\
\hline MetS & 0.601 & $0.517-0.685$ & 0.04 & 0.019 & 295.0 & $58.9 \%$ & $56.7 \%$ & 1.36 & 0.752 \\
\hline Obesity & 0.629 & $0.548-0.710$ & 0.04 & 0.004 & 295.0 & $58.5 \%$ & $61.8 \%$ & 1.53 & 0.67 \\
\hline Hypertension & 0.555 & $0.470-0.641$ & 0.04 & 0.203 & 295.0 & $58.8 \%$ & $55.8 \%$ & 1.33 & 0.73 \\
\hline High TG & 0.571 & $0.417-0.679$ & 0.05 & 0.144 & 295.0 & $57.4 \%$ & $51.1 \%$ & 1.17 & 0.83 \\
\hline Low HDL-C & 0.606 & $0.522-0.691$ & 0.04 & 0.015 & 295.0 & $58.1 \%$ & $58.7 \%$ & 1.40 & 0.71 \\
\hline High FBS & 0.663 & $0.566-0.759$ & 0.04 & 0.001 & 295.0 & $72.9 \%$ & $66.8 \%$ & 2.19 & 0.40 \\
\hline \multicolumn{10}{|l|}{ Men } \\
\hline MetS & 0.675 & $0.531-0.820$ & 0.07 & 0.030 & 310.5 & $66.7 \%$ & $61.9 \%$ & 1.75 & 0.54 \\
\hline Obesity & 0.763 & $0.626-0.901$ & 0.07 & 0.001 & 310.5 & $81.0 \%$ & $62.5 \%$ & 2.16 & 0.30 \\
\hline Hypertension & 0.636 & $0.486-0.787$ & 0.08 & 0.090 & 310.5 & $62.1 \%$ & $54.2 \%$ & 1.36 & 0.70 \\
\hline High TG & 0.798 & $0.652-0.944$ & 0.07 & 0.001 & 310.5 & $82.4 \%$ & $58.3 \%$ & 1.98 & 0.30 \\
\hline Low HDL-C & 0.783 & 0.661-0.905 & 0.06 & 0.001 & 310.5 & $84.2 \%$ & $61.8 \%$ & 2.20 & 0.26 \\
\hline High FBS & 0.536 & $0.377-0.696$ & 0.08 & 0.660 & 310.5 & $65.0 \%$ & $51.5 \%$ & 1.34 & 0.68 \\
\hline \multicolumn{10}{|l|}{ Women } \\
\hline MetS & 0.561 & $0.457-0.665$ & 0.05 & 0.237 & 275.0 & $58.3 \%$ & $44.8 \%$ & 1.06 & 0.93 \\
\hline Obesity & 0.659 & $0.566-0.753$ & 0.05 & 0.005 & 275.0 & $63.7 \%$ & $61.1 \%$ & 1.64 & 0.59 \\
\hline Hypertension & 0.504 & $0.398-0.609$ & 0.05 & 0.946 & 275.0 & $53.6 \%$ & $40.8 \%$ & 0.91 & 1.14 \\
\hline High TG & 0.451 & $0.323-0.579$ & 0.07 & 0.420 & 275.0 & $50.0 \%$ & $41.2 \%$ & 0.85 & 1.21 \\
\hline Low HDL-C & 0.608 & $0.505-0.711$ & 0.05 & 0.050 & 275.0 & $62.8 \%$ & $56.1 \%$ & 1.43 & 0.66 \\
\hline High FBS & 0.691 & $0.522-0.831$ & 0.07 & 0.002 & 275.0 & $75.0 \%$ & $49.5 \%$ & 1.49 & 0.51 \\
\hline
\end{tabular}

$S$ : standard error, SE: sensitivity, SP: specificity, LR+: positive likelihood ratio [=SE/(1-SP)], LR-: negative likelihood ratio $[=(1-S E) / S P]$.

Table 7.

The optimal cut-offs of clinical and laboratory variables in the prediction of MetS

\begin{tabular}{|c|c|c|c|c|c|c|c|c|c|}
\hline Variable & AUC & $95 \% \mathrm{CI}$ & $\mathrm{S}$ & $P$ & $\begin{array}{l}\text { Cut-off } \\
(\mathrm{mmol} / \mathrm{L})\end{array}$ & SE & SP & $\mathrm{LR}+$ & LR- \\
\hline Age, years & 0.634 & $0.551-0.716$ & 0.04 & 0.002 & 40.5 & $62.2 \%$ & $64.4 \%$ & 1.75 & 0.59 \\
\hline Weight, $\mathrm{Kg}$ & 0.693 & $0.615-0.771$ & 0.04 & $<0.001$ & 82.5 & $66.7 \%$ & $61.1 \%$ & 1.71 & 0.55 \\
\hline Height, $\mathrm{cm}$ & 0.590 & $0.507-0.673$ & 0.04 & 0.037 & 160.5 & $57.8 \%$ & $59.0 \%$ & 1.41 & 0.72 \\
\hline BMI, $\mathrm{kg} / \mathrm{m}^{2}$ & 0.689 & $0.609-0.769$ & 0.04 & $<0.001$ & 30.4 & $80.0 \%$ & $60.0 \%$ & 2.00 & 0.33 \\
\hline $\mathrm{UA}, \mathrm{mmol} / \mathrm{L}$ & 0.601 & $0.517-0.685$ & 0.04 & 0.019 & 295 & $58.9 \%$ & $56.7 \%$ & 1.36 & 0.72 \\
\hline $\mathrm{FBS}, \mathrm{mmol} / \mathrm{L}$ & 0.625 & $0.542-0.707$ & 0.04 & 0.004 & 5.5 & $52.2 \%$ & $73.3 \%$ & 1.96 & 0.65 \\
\hline HbA1c, \% & 0.673 & $0.594-0.752$ & 0.04 & $<0.001$ & 5.0 & $65.6 \%$ & $57.8 \%$ & 1.55 & 0.60 \\
\hline Insulin, $\mathrm{mlU} / \mathrm{mL}$ & 0.598 & $0.515-0.680$ & 0.04 & 0.024 & 4.29 & $54.4 \%$ & $53.4 \%$ & 1.17 & 0.85 \\
\hline HOMA-IR & 0.664 & $0.585-0.743$ & 0.04 & $<0.001$ & 1.23 & $56.7 \%$ & $63.3 \%$ & 1.54 & 0.68 \\
\hline $\mathrm{TC}, \mathrm{mmol} / \mathrm{L}$ & 0.633 & 0.551-0.715 & 0.04 & 0.002 & 4.97 & $62.2 \%$ & $60.0 \%$ & 1.56 & 0.63 \\
\hline $\mathrm{TG}, \mathrm{mg} / \mathrm{dL}$ & 0.666 & $0.587-0.745$ & 0.04 & $<0.001$ & 1.3 & $54.4 \%$ & $66.7 \%$ & 1.63 & 0.68 \\
\hline LDL-C, mmol/L & 0.648 & $0.565-0.730$ & 0.04 & 0.001 & 3.14 & $56.7 \%$ & $65.6 \%$ & 1.65 & 0.66 \\
\hline HDL-C, mmol/L & 0.514 & $0.429-0.598$ & 0.04 & 0.753 & 1.16 & $50.0 \%$ & $51.1 \%$ & 1.02 & 0.98 \\
\hline TC/HDL-C & 0.614 & $0.532-0.696$ & 0.04 & 0.008 & 4.2 & $61.1 \%$ & $60.0 \%$ & 1.53 & 0.65 \\
\hline TG/HDL-C & 0.660 & $0.580-0.741$ & 0.04 & $<0.001$ & 1.0 & $57.8 \%$ & $60.0 \%$ & 1.45 & 0.70 \\
\hline Non-HDL-C, mmol/L & 0.635 & $0.554-0.717$ & 0.04 & 0.002 & 3.8 & $60.0 \%$ & $64.4 \%$ & 1.69 & 0.62 \\
\hline
\end{tabular}

S: standard error, SE: sensitivity, SP: specificity, LR+: positive likelihood ratio [=SE/(1-SP)], LR-: negative likelihood ratio [=(1-SE)/SP]. 
The present study evaluated the associations of serum UA levels with MetS and its components in a sample of adult Saudi residence in the Northern area of Saudi Arabia. This region, as part of the "Middle East and North African (MENA)" region, is known for its high prevalence of MetS as recently supported by Al-Rubean et al.'s study, which revealed "a prevalence of $39.9 \%$ at specifically $45.0 \%$ in men and $35.4 \%$ in women" according to the same criteria the authors applied in the present study (i.e. NCEP ATP III criteria). ${ }^{(16)}$ The authors preferred to apply the latter criteria rather than that of the International Diabetes Federation (IDF) (23) which mandates the presence of central obesity as one of the MetS components, contributing to apparently less disease prevalence and missing of several risky individuals who have other MetS components.

Currently, participants older than 45 years showed three times the odds of having MetS compared with those less than 45 years old. This could be attributed in part to the association of the age with the increased central obesity, hormonal changes, and IR, ${ }^{(24,25)}$ as well as the analogous increase in the prevalence of specific MetS components (e.g. diabetes and hypertension) with age in the current population. . $^{(26,27)}$

In the present study, the serum UA level was higher in the MetS group compared to non-MetS one. This finding was consistent with the previous studies in other areas of the world. ${ }^{(28-32)}$ Although serum UA has been reported to have a potent antioxidant capacity (i.e. conferring nearly $50 \%-60 \%$ of the total plasma antioxidant activity) in the circulation, ${ }^{(33)}$ it can promote oxidative and inflammatory stress in the intracellular context $^{(34-36)}$ by several mechanisms detailed previously ${ }^{(37-39)}$ which lead to endothelial cell dysfunction and contribute to MetS biogenesis. ${ }^{(34-36)}$

The overall analysis revealed that participants in the fourth quartile of serum UA showed a significantly higher frequency of MetS and central obesity relative to the ones in the first quartiles. This finding could support the mutual relations that could be present between serum UA and MetS/ components, which have been debated recently. ${ }^{(40)}$ Chronic hyperuricemia found to be implicated in the pathogenesis of metabolic perturbation that could lead to MetS. Its control may prevent or reverse the course of MetS and/or its components. ${ }^{(41,42)}$ Previously, Zhang et al.'s longitudinal cohort study, and a study by Chen et al. revealed that individuals with a higher concentration of serum UA, have 1.6 times the risk of developing MetS. ${ }^{(43,44)}$ Furthermore, Nejatinamini et al. showed that higher serum UA levels, "even within the normal ranges", were associated with increased odds to have MetS (nearly double risk per one unit increment in serum UA), and could be considered as one of the determinants of the MetS. ${ }^{(31)}$ Our findings are also consistent with that of Choi and Ford 's study on a nationally-representative sample of US adults. ${ }^{(45)}$ They found that MetS prevalence and its individual components increase with increasing grades of hyperurecemia, and they recommended more intensive clinical investigation for a potential coexistence of the MetS in cases presented with high serum UA levels. ${ }^{(4)}$ The close relation of hyperuricemia with MetS might be supported by the fact that UA biosynthesis is linked to glycolysis that is regulated by insulin. A decrease in the glycolytic enzyme glyceraldehyde3-phosphate dehydrogenase (GA3PDH) activity in case of insulin resistance leads to shifting of glucose utilization from glycolysis into other pathways like the pentose phosphate pathway which is the main source of ribose-5-phosphate, the building unit of phosphoribosyl pyrophosphate (PRPP), which participates in the synthesis of nucleic acids including the purines and, consequently, increases its degradation products and the emergence of hyperuricemia. ${ }^{(46)}$ In this respect, the latter mechanism could also explain the current finding of the high frequency of central obesity in high UA level quartiles as it is known that the visceral fat to be specifically associated with more insulin resistance than other types of adiposity due to the combined effect of increase free fatty acids released and adipokine secretion deregulation. ${ }^{(47)}$

Although overall correlation analysis showed that serum UA was correlated with age, BMI, and abdominal obesity, stratification analysis by sex revealed a consistent correlation of both BMI and central obesity with serum UA in both sexes. Still, a significant correlation with age was only evident in females. Interestingly, this finding is in line with recent findings of Sun et al., who revealed correlations between UA, hyperuricemia, and coronary artery diseases only in females, but not in men. ${ }^{(48)}$ Additionally, several previous studies in different areas of the world supported this association like the National Health and Nutrition Examination Survey (NANHES) on the US general population, ${ }^{(49)}$ the Italian Pro. V. A. study ${ }^{(50)}$ and the community-based study in China. ${ }^{(51)}$ Although the exact cause of this association is still undefined, the postmenopausal decline of the protective estrogens might explain in part this relation and the consistent correlation of serum UA with the glycemic variables (FBS and $\mathrm{HbA1c} \%$ ) in the present enrolled women. ${ }^{(52)}$

The present study identified an inverse correlation between serum UA and HDL-C levels and significant positive correlations between serum UA and other laboratory parameters, including the lipid profile-related variables and ratios (TC/HDL-C, TG/ HDL-C, non-HDL-C, and TG/HDL-C) in overall analysis with consistent associations with HDL-C and TG/HDL in both men and women after stratification by sex. This finding expands the previous studies that correlate serum UA with dyslipidemia and an increase in the risk of developing high LDL-C and hypertriglyceridemia. ${ }^{(53,54)}$ The TG/HDL-C ratio is known to be a practical approach for identifying individuals who have insulin resistance, as concluded previously. ${ }^{(55,56)}$ Given the role of HDL-C in the reverse transport of cholesterol from the peripheral tissues to the liver, the inhibitory effect on LDL oxidation, attenuation of platelet aggregation, and stimulation of prostacyclin secretion, HDL-C has a protective role against atherogenicity. ${ }^{(57)}$ As previously indicated that it is hard to estimate the "LDL particle size" increment, which is associated with the hypertriglyceridemia risk, ${ }^{(58)} \mathrm{TG} /$ HDL ratio is considered an accurate and putative marker for atherogenicity, ${ }^{(55)}$ and prediction of the cardio-metabolic risk. ${ }^{(59,60)}$

Remarkably, on testing the predictive capacity of serum UA to predict MetS and/or its components, the current results were largely in line with the available literature concerning 
adult subjects. ${ }^{(61,62)}$ These studies including ours, suggest that hyperuricemic individuals could develop MetS at specified cut-off values among different sexes. It is noteworthy that these cut-off values $(310 \mu \mathrm{mol} / \mathrm{L}$ and $275.0 \mu \mathrm{mol} / \mathrm{L}$ in the enrolled men and women, respectively) are less than those proposed for the gout treatment ${ }^{(63)}$, which can decrease the chance of missing individuals with high risk to cardiovascular diseases (including MetS individuals) as supported by Cicero et al. ${ }^{(62)}$ Although overall serum UA discriminating value was not the best one among other predictors for MetS, its cut-off point was associated with moderate sensitivity and specificity values, which minimize the false-positive and the falsenegative included cases.

Collectively, it is evident that there is a significant association of serum UA levels with MetS and its components, including those associated with an increase in the risk of cardiovascular diseases. At specific cut-off levels, serum UA might predict MetS and/or its components in a sex-specific pattern in the study population that deserves great concern for careful monitor and control. It is worth noting that some limitations should be considered. The study design is a crosssectional with a modest sample size that enrolled patients with relatively health awareness who were routinely attending the Medicine outpatient clinics at the general hospital, which could add some source of selection bias as it does not represent the general population. Not all the confounding factors could be adjusted (e.g. the physical activity, nutritional status, etc.) as the related data were not complete. Also, the direct causal relation of UA with MetS could not infer from the current study. Longitudinal follow-up studies with larger sample size and with different ethnicities are recommended.

\section{Conclusion}

The study findings suggest that levels of serum UA in the Saudi population might be associated with the risk of MetS and its components. In this sense, modifying the lifestyle and early management of hyperuricemia could be a useful strategy for lowering the MetS burden in this region. It is highly recommended to explore the causal relationship between hyperuricemia and MetS in future large-cohort studies.

\section{Competing Interests} interests.

The authors declare that they have no competing

\section{Sources of Funding}

This research project was supported by a grant from the "Deanship of Scientific Research, Northern Border University (NBU), Arar, Saudi Arabia” (MED-2017-1-7-F-7140).

\section{References}

1. Feig DI, Madero M, Jalal DI, Sanchez-Lozada LG, Johnson RJ. Uric acid and the origins of hypertension. J Pediatr. 2013;162(5):896-902. doi:10.1016/j.jpeds.2012.12.078
2. Neogi T, George J, Rekhraj S, Struthers AD, Choi $\mathrm{H}$, Terkeltaub RA. Are either or both hyperuricemia and xanthine oxidase directly toxic to the vasculature? A critical appraisal. Arthritis Rheum. 2012;64(2):327-338. doi:10.1002/ art.33369

3. Gagliardi AC, Miname MH, Santos RD. Uric acid: A marker of increased cardiovascular risk. Atherosclerosis. 2009;202(1):11-17. doi:10.1016/j.atherosclerosis.2008.05.022 4. Soltani Z, Rasheed K, Kapusta DR, Reisin E. Potential role of uric acid in metabolic syndrome, hypertension, kidney injury, and cardiovascular diseases: is it time for reappraisal?. Curr Hypertens Rep. 2013;15(3):175-181. doi:10.1007/s11906-013-0344-5

5. Tamariz L, Agarwal S, Soliman EZ, Chamberlain AM, Prineas R, Folsom AR, et al. Association of serum uric acid with incident atrial fibrillation (from the Atherosclerosis Risk in Communities [ARIC] study). Am J Cardiol. 2011;108(9):1272-1276. doi:10.1016/j.amjcard.2011.06.043

6. Liu P, Jiang Y, Meng J. Relationship between serum uric acid, metabolic syndrome and resting heart rate in Chinese elderly. Obes Res Clin Pract. 2016;10(2):159-168. doi:10.1016/j.orcp.2015.04.007

7. Suzuki I, Yamauchi T, Onuma M, Nozaki S. Allopurinol, an inhibitor of uric acid synthesis--can it be used for the treatment of metabolic syndrome and related disorders?. Drugs Today (Barc). 2009;45(5):363-378. doi:10.1358/dot.2009.45.5.1370460

8. DeBosch BJ, Kluth O, Fujiwara H, Schürmann A, Moley K. Early-onset metabolic syndrome in mice lacking the intestinal uric acid transporter SLC2A9. Nat Commun. 2014;5:4642. Published 2014 Aug 7. doi:10.1038/ncomms5642

9. Yuan H, Yu C, Li X, Sun L, Zhu X, Zhao C, et al. Serum Uric Acid Levels and Risk of Metabolic Syndrome: A DoseResponse Meta-Analysis of Prospective Studies. J Clin Endocrinol Metab. 2015;100(11):4198-4207. doi:10.1210/ jc.2015-2527

10. Fawzy MS, Abu AlSel BT. Assessment of Vitamin D-Binding Protein and Early Prediction of Nephropathy in Type 2 Saudi Diabetic Patients. J Diabetes Res. 2018;2018:8517929. Published 2018 Apr 3. doi:10.1155/2018/8517929

11. Fawzy MS, Alshammari MA, Alruwaili AA, et al. Factors associated with diabetic foot among type 2 diabetes in Northern area of Saudi Arabia: a descriptive study. BMC Res Notes. 2019;12(1):51. Published 2019 Jan 22. doi:10.1186/ s13104-019-4088-4

12. American Heart Association; National Heart, Lung, and Blood Institue, Grundy SM, et al. Diagnosis and management of the metabolic syndrome. An American Heart Association/ National Heart, Lung, and Blood Institute Scientific Statement. Executive summary. Cardiol Rev. 2005;13(6):322-327.

13. Al-Rubean K, Youssef AM, AlFarsi Y, Al-Sharqawi AH, Bawazeer N, AlOtaibi MT, et al. Anthropometric cutoff values for predicting metabolic syndrome in a Saudi community: from the SAUDI-DM study. Ann Saudi Med. 2017;37(1):2130. doi:10.5144/0256-4947.2017.21

14. Scott R, Donoghoe M, Watts GF, O’Brien R, Pardy C, Taskinen MR, et al. Impact of metabolic syndrome and its components on cardiovascular disease event rates in 4900 patients with type 2 diabetes assigned to placebo in the FIELD randomised trial. Cardiovasc Diabetol. 2011;10:102. Published 2011 Nov 21. doi:10.1186/1475-2840-10-102 15. Huang PL. A comprehensive definition for metabolic syndrome. Dis Model Mech. 2009;2(5-6):231-237. doi:10.1242/dmm.001180 
16. Al-Rubeaan K, Bawazeer N, Al Farsi Y, et al. Prevalence of metabolic syndrome in Saudi Arabia - a cross sectional study. BMC Endocr Disord. 2018;18(1):16. Published 2018 Mar 5. doi:10.1186/s12902-018-0244-4

17. Chu SY, Jung JH, Park MJ, Kim SH. Risk assessment of metabolic syndrome in adolescents using the triglyceride/ high-density lipoprotein cholesterol ratio and the total cholesterol/high-density lipoprotein cholesterol ratio. Ann Pediatr Endocrinol Metab. 2019;24(1):41-48. doi:10.6065/ apem.2019.24.1.41

18. Ghodsi S, Meysamie A, Abbasi M, et al. Non-highdensity lipoprotein fractions are strongly associated with the presence of metabolic syndrome independent of obesity and diabetes: a population-based study among Iranian adults. J Diabetes Metab Disord. 2017;16:25. Published 2017 Jun 7. doi:10.1186/s40200-017-0306-6

19. Bonora E, Formentini G, Calcaterra F, Lombardi S, Marini F, Zenari L, et al. HOMA-estimated insulin resistance is an independent predictor of cardiovascular disease in type 2 diabetic subjects: prospective data from the Verona Diabetes Complications Study. Diabetes Care. 2002;25(7):1135-1141. doi:10.2337/diacare.25.7.1135

20. Vogeser M, König D, Frey I, Predel HG, Parhofer KG, Berg A. Fasting serum insulin and the homeostasis model of insulin resistance (HOMA-IR) in the monitoring of lifestyle interventions in obese persons. Clin Biochem. 2007;40(1314):964-968. doi:10.1016/j.clinbiochem.2007.05.009

21. NCD Risk Factor Collaboration (NCD-RisC). Worldwide trends in body-mass index, underweight, overweight, and obesity from 1975 to 2016: a pooled analysis of 2416 populationbased measurement studies in 128.9 million children, adolescents, and adults. Lancet. 2017;390(10113):2627-2642. doi:10.1016/S0140-6736(17)32129-3

22. Danaei G, Finucane MM, Lu Y, Singh GM, Cowan MJ, Paciorek CJ, et al. National, regional, and global trends in fasting plasma glucose and diabetes prevalence since 1980: systematic analysis of health examination surveys and epidemiological studies with 370 country-years and $2 \cdot 7$ million participants. Lancet. 2011;378(9785):31-40. doi:10.1016/S0140-6736(11)60679-X

23. Alberti KG, Zimmet P, Shaw J. Metabolic syndrome-a new world-wide definition. A Consensus Statement from the International Diabetes Federation. Diabet Med. 2006;23(5):469-480. doi:10.1111/j.1464-5491.2006.01858.x 24. Boden G, Chen X, DeSantis RA, Kendrick Z. Effects of age and body fat on insulin resistance in healthy men. Diabetes Care. 1993;16(5):728-733. doi:10.2337/diacare.16.5.728

25. Park YW, Zhu S, Palaniappan L, Heshka S, Carnethon MR, Heymsfield SB. The metabolic syndrome: prevalence and associated risk factor findings in the US population from the Third National Health and Nutrition Examination Survey, 1988-1994. Arch Intern Med. 2003;163(4):427-436. doi:10.1001/archinte.163.4.427

26. Al-Nozha MM, Abdullah M, Arafah MR, Khalil MZ, Khan NB, Al-Mazrou YY, et al. Hypertension in Saudi Arabia. Saudi Med J. 2007;28(1):77-84.

27. Al-Rubeaan K, Al-Manaa H, Khoja T, Ahmad N, AlSharqawi A, Siddiqui K, et al. The Saudi Abnormal Glucose Metabolism and Diabetes Impact Study (SAUDI-DM). Ann Saudi Med. 2014;34(6):465-475. doi:10.5144/02564947.2014.465

28. Reimann M, Schutte AE, Malan L, Huisman HW, Malan NT. Hyperuricaemia is an independent factor for the metabolic syndrome in a sub-Saharan African population: a factor analysis. Atherosclerosis. 2008;197(2):638-645. doi:10.1016/j.atherosclerosis.2007.09.011

29. Sui X, Church TS, Meriwether RA, Lobelo F, Blair $\mathrm{SN}$. Uric acid and the development of metabolic syndrome in women and men. Metabolism. 2008;57(6):845-852. doi:10.1016/j.metabol.2008.01.030

30. Numata T, Miyatake N, Wada J, Makino H. Comparison of serum uric acid levels between Japanese with and without metabolic syndrome. Diabetes Res Clin Pract. 2008;80(1):e1e5. doi:10.1016/j.diabres.2007.10.031

31. Nejatinamini S, Ataie-Jafari A, Qorbani M, Nikoohemat $\mathrm{S}$, Kelishadi R, Asayesh H, et al. Association between serum uric acid level and metabolic syndrome components. J Diabetes Metab Disord. 2015;14:70. Published 2015 Sep 14. doi:10.1186/s40200-015-0200-z

32. Gonçalves JP, Oliveira A, Severo M, Santos AC, Lopes C. Cross-sectional and longitudinal associations between serum uric acid and metabolic syndrome. Endocrine. 2012;41(3):450457. doi:10.1007/s12020-012-9629-8

33. Schumacher HR Jr, Becker MA, Wortmann RL, et al. Effects of febuxostat versus allopurinol and placebo in reducing serum urate in subjects with hyperuricemia and gout: a 28-week, phase III, randomized, double-blind, parallel-group trial. Arthritis Rheum. 2008;59(11):1540-1548. doi:10.1002/art.24209

34. Furukawa S, Fujita T, Shimabukuro M, Iwaki M, Yamada Y, Nakajima Y, et al. Increased oxidative stress in obesity and its impact on metabolic syndrome. J Clin Invest. 2004;114(12):1752-1761. doi:10.1172/JCI21625

35. Cheung KJ, Tzameli I, Pissios P, Rovira I, Gavrilova $\mathrm{O}$, Ohtsubo T, et al. Xanthine oxidoreductase is a regulator of adipogenesis and PPARgamma activity. Cell Metab. 2007;5(2):115-128. doi:10.1016/j.cmet.2007.01.005

36. Sautin YY, Johnson RJ. Uric acid: the oxidantantioxidant paradox. Nucleosides Nucleotides Nucleic Acids. 2008;27(6):608-619. doi:10.1080/15257770802138558

37. Kanbay M, Jensen T, Solak Y, Le M, Roncal-Jimenez $\mathrm{C}$, Rivard $\mathrm{C}$, et al. Uric acid in metabolic syndrome: From an innocent bystander to a central player. Eur J Intern Med. 2016;29:3-8. doi:10.1016/j.ejim.2015.11.026

38. Battelli MG, Bortolotti M, Polito L, Bolognesi A. The role of xanthine oxidoreductase and uric acid in metabolic syndrome. Biochim Biophys Acta Mol Basis Dis. 2018;1864(8):2557-2565. doi:10.1016/j.bbadis.2018.05.003

39. Roumeliotis S, Roumeliotis A, Dounousi E, Eleftheriadis T, Liakopoulos V. Dietary Antioxidant Supplements and Uric Acid in Chronic Kidney Disease: A Review. Nutrients. 2019;11(8):1911. Published 2019 Aug 15. doi:10.3390/ nu11081911

40. De Pergola G, Cortese F, Termine G, Meliota G, Carbonara R, Masiello M, et al. Uric Acid, Metabolic Syndrome and Atherosclerosis: The Chicken or the Egg, Which Comes First?. Endocr Metab Immune Disord Drug Targets. 2018;18(3):251-259. doi:10.2174/18715303186661 80212101548

41. Bombelli M, Quarti-Trevano F, Tadic M, Facchetti R, Cuspidi C, Mancia G, et al. Uric acid and risk of new-onset metabolic syndrome, impaired fasting glucose and diabetes mellitus in a general Italian population: data from the Pressioni Arteriose Monitorate E Loro Associazioni study. J Hypertens. 2018;36(7):1492-1498. doi:10.1097/HJH.0000000000001721 42. King C, Lanaspa MA, Jensen T, Tolan DR, SánchezLozada LG, Johnson RJ. Uric Acid as a Cause of the 
Metabolic Syndrome. Contrib Nephrol. 2018;192:88-102. doi: $10.1159 / 000484283$

43. Chen LY, Zhu WH, Chen ZW, Dai HL, Ren JJ, Chen $\mathrm{JH}$, et al. Relationship between hyperuricemia and metabolic syndrome. J Zhejiang Univ Sci B. 2007;8(8):593-598. doi:10.1631/jzus.2007.B0593

44. Zhang Q, Zhang C, Song X, Lin H, Zhang D, Meng W, et al. A longitudinal cohort based association study between uric acid level and metabolic syndrome in Chinese Han urban male population. BMC Public Health. 2012;12:419. Published 2012 Jun 8. doi:10.1186/1471-2458-12-419

45. Choi HK, Ford ES. Prevalence of the metabolic syndrome in individuals with hyperuricemia. Am J Med. 2007;120(5):442-447. doi:10.1016/j.amjmed.2006.06.040

46. Matsuura F, Yamashita S, Nakamura T, et al. Effect of visceral fat accumulation on uric acid metabolism in male obese subjects: visceral fat obesity is linked more closely to overproduction of uric acid than subcutaneous fat obesity. Metabolism. 1998;47(8):929-933. doi:10.1016/ s0026-0495(98)90346-8

47. Westphal SA. Obesity, abdominal obesity, and insulin resistance. Clin Cornerstone. 2008;9(1):23-31. doi:10.1016/ s1098-3597(08)60025-3

48. Sun Y, Zhang H, Tian W, Shi L, Chen L, Li J, et al. Association between serum uric acid levels and coronary artery disease in different age and gender: a cross-sectional study. Aging Clin Exp Res. 2019;31(12):1783-1790. doi:10.1007/s40520-019-01137-2

49. Zhu Y, Pandya BJ, Choi HK. Prevalence of gout and hyperuricemia in the US general population: the National Health and Nutrition Examination Survey 2007-2008. Arthritis Rheum. 2011;63(10):3136-3141. doi:10.1002/art.30520

50. Musacchio E, Perissinotto E, Sartori L, Veronese N, Punzi L, Zambon S, et al. Hyperuricemia, Cardiovascular Profile, and Comorbidity in Older Men and Women: The Pro.V.A. Study. Rejuvenation Res. 2017;20(1):42-49. doi:10.1089/ rej.2016.1834

51. Huang G, Xu JB, Zhang TJ, Nie XL, Li Q, Liu Y, et al. Hyperuricemia is associated with cardiovascular diseases clustering among very elderly women - a community based study in Chengdu, China. Sci Rep. 2017;7(1):996. Published 2017 Apr 20. doi:10.1038/s41598-017-01042-6

52. Wallace IR, McKinley MC, Bell PM, Hunter SJ. Sex hormone binding globulin and insulin resistance. Clin Endocrinol (Oxf). 2013;78(3):321-329. doi:10.1111/ cen. 12086

53. Peng TC, Wang CC, Kao TW, Chan JY, Yang YH, Chang YW, et al. Relationship between hyperuricemia and lipid profiles in US adults. Biomed Res Int. 2015;2015:127596. doi:10.1155/2015/127596
54. Kuwabara M, Borghi C, Cicero AFG, Hisatome I, Niwa $\mathrm{K}$, Ohno M, et al. Elevated serum uric acid increases risks for developing high LDL cholesterol and hypertriglyceridemia: A five-year cohort study in Japan. Int J Cardiol. 2018;261:183188. doi:10.1016/j.ijcard.2018.03.045

55. González-Chávez A, Simental-Mendía LE, ElizondoArgueta S. Elevated triglycerides/HDL-cholesterol ratio associated with insulin resistance. Cir Cir. 2011;79(2):126131.

56. Salazar MR, Carbajal HA, Espeche WG, Leiva Sisnieguez CE, March CE, Balbin E, et al. Comparison of the abilities of the plasma triglyceride/high-density lipoprotein cholesterol ratio and the metabolic syndrome to identify insulin resistance. Diab Vasc Dis Res. 2013;10(4):346-352. doi:10.1177/1479164113479809

57. Toth PP. High-density lipoprotein and cardiovascular risk. Circulation. 2004;109(15):1809-1812. doi:10.1161/01. CIR.0000126889.97626.B8

58. Maruyama C, Imamura $\mathrm{K}$, Teramoto $\mathrm{T}$. Assessment of LDL particle size by triglyceride/HDL-cholesterol ratio in nondiabetic, healthy subjects without prominent hyperlipidemia. J Atheroscler Thromb. 2003;10(3):186-191. doi:10.5551/ jat. 10.186

59. Salazar MR, Carbajal HA, Espeche WG, Leiva Sisnieguez CE, Balbin E, Dulbecco CA, et al. Relation among the plasma triglyceride/high-density lipoprotein cholesterol concentration ratio, insulin resistance, and associated cardio-metabolic risk factors in men and women. Am J Cardiol. 2012;109(12):17491753. doi:10.1016/j.amjcard.2012.02.016

60. Salazar MR, Carbajal HA, Espeche WG, Aizpurua M, Leiva Sisnieguez CE, March CE, et al. Identifying cardiovascular disease risk and outcome: use of the plasma triglyceride/high-density lipoprotein cholesterol concentration ratio versus metabolic syndrome criteria. J Intern Med. 2013;273(6):595-601. doi:10.1111/joim.12036

61. Zurlo A, Veronese N, Giantin V, Maselli M, Zambon S, Maggi S, et al. High serum uric acid levels increase the risk of metabolic syndrome in elderly women: The PRO.V.A study. Nutr Metab Cardiovasc Dis. 2016;26(1):27-35. doi:10.1016/j.numecd.2015.10.007

62. Cicero AFG, Fogacci F, Giovannini M, Grandi E, Rosticci M, D'Addato S, et al. Serum uric acid predicts incident metabolic syndrome in the elderly in an analysis of the Brisighella Heart Study. Sci Rep. 2018;8(1):11529. Published 2018 Aug 1. doi:10.1038/s41598-018-29955-w

63. Richette P, Doherty M, Pascual E, Barskova V, Becce F, Castaneda-Sanabria J, et al. 2016 updated EULAR evidence-based recommendations for the management of gout. Ann Rheum Dis. 2017;76(1):29-42. doi:10.1136/ annrheumdis-2016-209707 